\title{
Índice de autores 2020
}

La Revista de la Asociación Argentina de Ortopedia y Traumatología agradece a los siguientes autores por habernos elegido para publicar sus trabajos. Esperamos seguir contando con su valiosa colaboración.

Accoce M. ...................................................................... 357

Adriazola Gallardo F. ....................................................... 56

Aguer S.

Aguirre G. L. ................................................................. 11

Alberghini M. .................................................................... 246

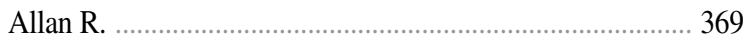

Allende Nores C. ...................................................... 11; 246

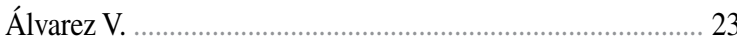

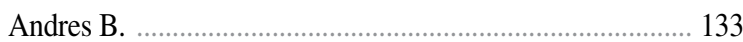

Aparicio J. L. ............................................................... 234

Argüelles S.

Arzac Ulla I.

Autorino C. M.

Ayerza M. A.

Baldo B.

Barbero M.

Bazán P. L.

$31 ; 119$

Becerra Orrego M. O.

Bennice J.

Beraún Coronel L. A.

Beribé R.

Bersusky E. 377

Bertona Altieri A.

Betemps A.

Bidolegui F.

Biolatto P.

$65 ; 418$

Bizzarri P.

Bochatey E. J.

Bordachar D.

Boretto J. G.

$125 ; 325$

Borri Á. E

31; 119

Bronenberg Victorica P.

Caloia M. S36

Calvi J. P.
Calvo A. B. 23

Cancinos J. D. 31

Carabajal Mattar M. 418

Carlucci S. 305

Carrasco M. 305

Carrioli G. 283

Cattáneo A.

Caviglia H. A. 262

Cervigni F. S19

Chen Tai Lo. 357

Ciccioli N. M.

Colesnik P. 357

Conti L.

Contreras V. A. 349

Coombes N.

Coppola E. F.

Cosini F. N.

Costa Paz M. .74

Cristiani M.

Cubecino A.

Cucchiara J. P.

Dal Lago J. E.

De Carli P.

de Zavalía M.

Deimundo $\mathrm{M}$.

del Sel H.

107; 317

Dell'Era S.

Díaz R.

Di Memmo D.

Dimaro M.

Donndorff A.

Dozo C.

Eljatib A. 


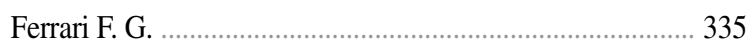

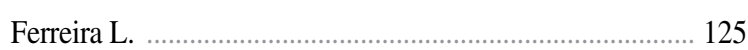

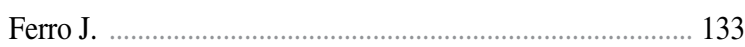

Fiminella H. .............................................................. 133

Francheri Wilson I. A. .................................................. 377

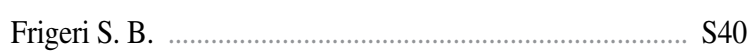

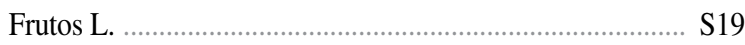

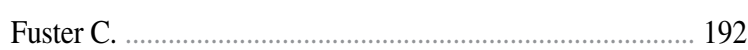

Gaitan L. C. ..................................................................... 23

Galaretto E. ........................................................ 192; 377

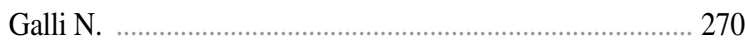

Gallucci G. L. ................................................. 125; 325

Gamarra D. .............................................................. 437

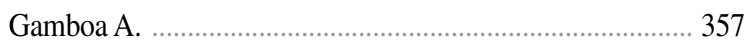

Garabano G.

$107 ; 317 ; 437$

Garay A. L.

133; S12

García F. ........................................................................ 270

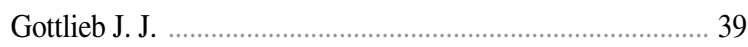

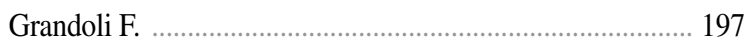

Grasso B. .............................................................. S19

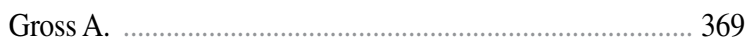

Gutiérrez Olivera N. ......................................................... 246

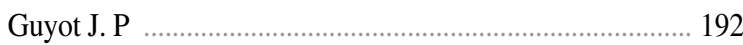

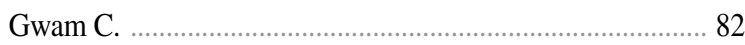

Hernandez D. .................................................................. 357

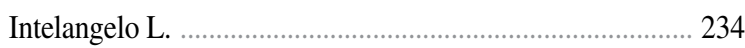

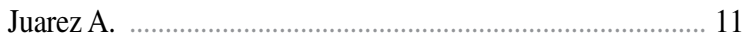

Juárez Cesca F. .................................................................. 204

Lasser D. ................................................................... S40

Layús M. ......................................................................... 2

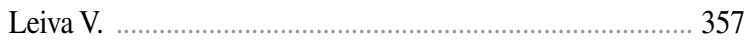

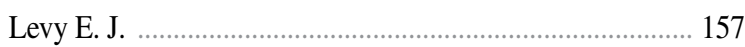

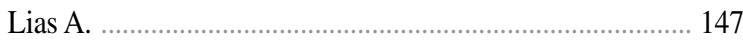

Lobos E. .......................................................................... 11

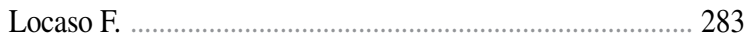

López Barbieri S. ............................................................... 56

López Bustos F. ……………….......................... 133; S12

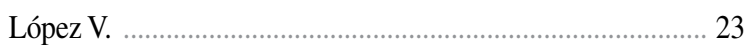

Lopreite F. .................................................. 107; 214; 317

Loterzo L.G. .................................................................... 301

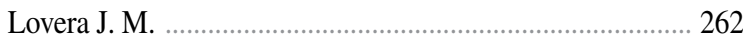

Lucioni F.

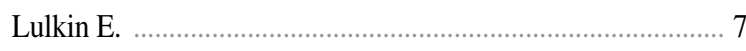

Macchiavello Falcon R. …………………………….......... 393

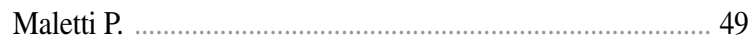

Marín E. ........................................................................... 119

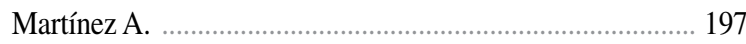

Martínez C. E. ………………................................ 133; S12

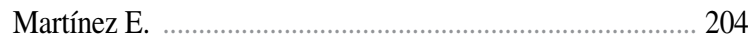

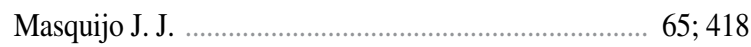

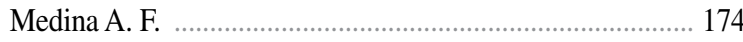

Medina M. ................................................................... 119

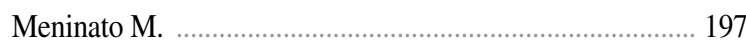

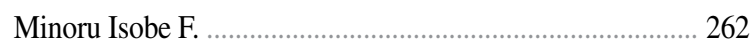

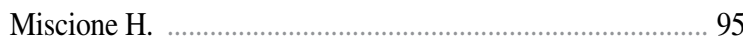

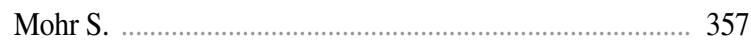

Molina Rómoli A. R. ........................................................ 82

Morales Vásquez E. ......................................................... 393

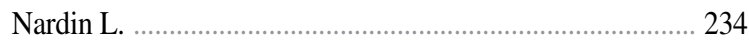

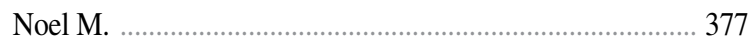

Nuñez Alvarado L. E. ……………………………............ 393

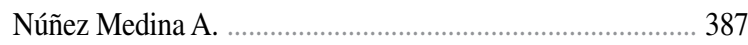

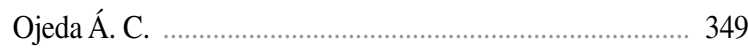

Olivetto J. M. …………….................................................. 437

Orellana C. ...................................................................... 377

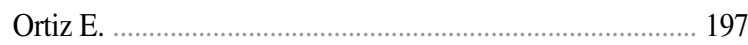

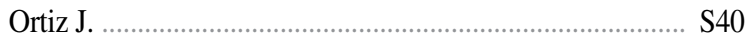

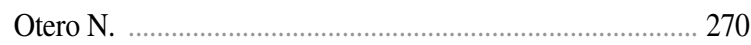

Pachón Guzmán A. ……………................................... 174

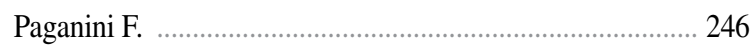

Paladino L. ....................................................................... 369

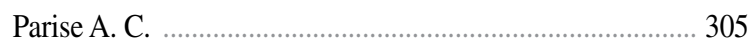

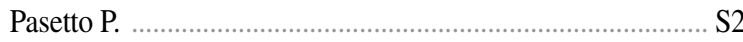

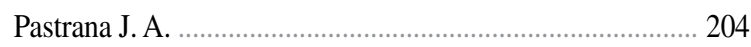

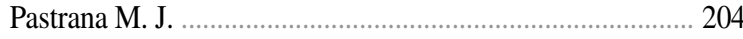

Patiño J. M. …………............................................ 133; S12

Patiño O. ……................................................ 74; 234

Pereira E. ........................................................ 335; 403; S1

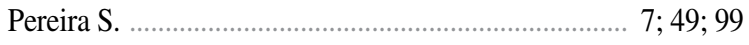

Pérez M. ........................................................................ 283

Pérez Alamino L. ............................................................. 107

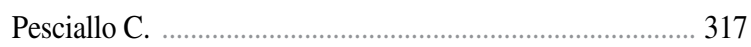

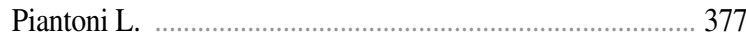

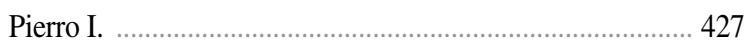


Piuzzi N. S.

Praglia F.

222

Quispe Juárrez J. G. 254

Quiroga R. .. 151

Racca M. S19

Rasumoff A. 167

Reble J. 119

Reinares F. ... 335

Rellán I. 125

Remondino R. .. 377

Resano H. 39

Ricciardi D ... 283

Ricciardi G. 283

Ricciardi G. A. ... 283

Rivas E. 369

Robador N. ... 437

Robles C. .. 11

Rodríguez-López T. 387

Rodríguez M. P. 403

Rodríguez R. 357

Rodriguez Sammartino M. .............................................. 187

Román E. ... 147

Romano O. 147

Romano Yalour N.

.. 31

Rossi L. A. .. 82

Ruiz C. ... 133

Saidman J. M. 167

Salas J. M. .. 139

Sánchez Saba J. E. 74

Sánchez Villanueva F. J.

Santini Araujo G.

Sanz M.

Sanzana E. S.

Saprizza S.

Scalambro S.
Seré I. 197

Sierto M. 437

Silberman F. S.

Simesen de Bielke H.

107; 437

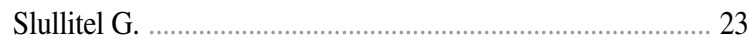

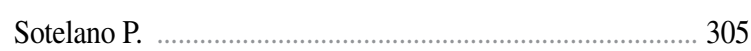

Soutullo J. L .................................................................... 403

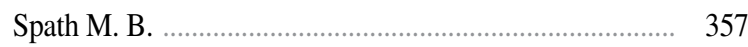

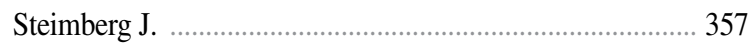

Tálamo F. ...................................................................... 427

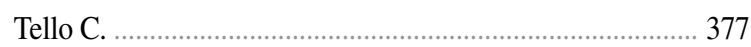

Terpolilli E. .................................................................... 357

Tonetto G. ................................................................... S12

Trueba R. ......................... 3; 91; 97; 184; 190; 295; 303; 447

Turazza F. .................................................................... 418

Valle P. ................................................................... S19

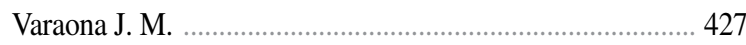

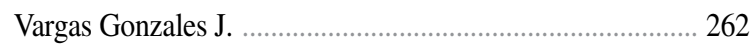

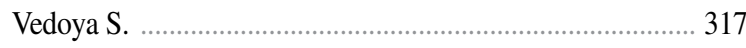

Velasco Canziani J. M. ....................................................... 270

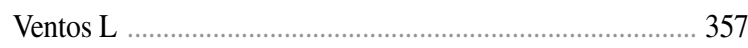

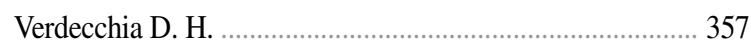

Viale G. ………………................................................... 317

Villena D. S. ....................................................................... 305

Vindver G. ..................................................... 7; 49 ; 99

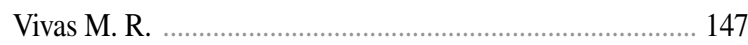

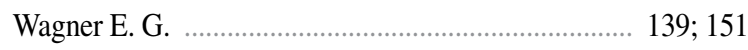

Williams E. ..................................................................... 357

Yacuzzi C. ……….............................................................. 74

Zaidenberg C. R. ................................................. 204; 335

Zaidenberg E. E. ..................................... 125; 204; 325; S26

Zancolli E. A. ............................................................ S45

Zancolli E. P. …........................................................... S45

Zancolli E. R. ................................................... S36; S48

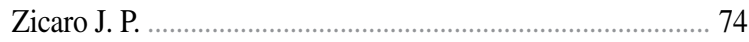

